\title{
Thoracic involvement and imaging patterns in IgG4-related disease
}

\author{
Romain Muller $\mathbb{1}^{1,13}$, Paul Habert ${ }^{2,13}$, Mikael Ebbo ${ }^{1}$, Julie Graveleau ${ }^{3}$, Mathieu Groh ${ }^{4}$, David Launay $\mathbb{C}^{5}$, \\ Sylvain Audia ${ }^{6}$, Gregory Pugnet ${ }^{7}$, Fleur Cohen $^{8}$, Antoinette Perlat ${ }^{9}$, Audrey Benyamine ${ }^{10}$, Boris Bienvenu ${ }^{11}$, \\ Lea Gaigne ${ }^{1}$, Pascal Chanez ${ }^{12}$, Jean Yves Gaubert ${ }^{2}$ and Nicolas Schleinitz (1) ${ }^{1}$
}

\begin{abstract}
${ }^{1}$ Dept of Internal Medicine, Aix Marseille University, APHM, CHU La Timone, Marseille, France. ${ }^{2}$ Dept of Radiology, Aix Marseille University, APHM, CHU La Timone, Marseille, France. ${ }^{3}$ Dept of Internal Medicine, CH Saint Nazaire, Saint Nazaire, France. ${ }^{4}$ Dept of Internal Medicine, CH Foch, National Referral Center for Hypereosinophilic Syndromes (CEREO), Suresnes, France. ${ }^{5}$ Univ. Lille, Inserm, CHU Lille, Service de Médecine Interne et Immunologie Clinique, Centre de référence des maladies autoimmunes systémiques rares du Nord et Nord-Ouest de France (CeRAINO), U1286 - INFINITE - Institute for Translational Research in Inflammation, Lille, France. ${ }^{6}$ Dept of Internal Medicine, CHU Dijon, Dijon, France. ${ }^{7}$ Dept of Internal Medicine and Clinical Immunology, CHU Toulouse, Toulouse, France. ${ }^{8}$ Dept of Internal Medicine, APHP, CHU Pitié-Salpêtrière, Paris, France. ${ }^{9}$ Dept of Internal Medicine, CHU Rennes, Rennes, France. ${ }^{10}$ Dept of Internal Medicine, Aix Marseille University, APHM, CHU Nord, Marseille, France. ${ }^{11}$ Dept of Internal Medicine, CH St Joseph, Marseille, Marseille, France. ${ }^{12}$ Dept of Pneumology, Aix Marseille University, APHM, CHU Nord, Marseille, France. ${ }^{13}$ These authors contributed equally to this work.
\end{abstract}

Corresponding author: Nicolas Schleinitz (nicolas.schleinitz@ap-hm.fr)

Shareable abstract (@ERSpublications)

Thoracic and lung involvements of IgG4-RD are heterogeneous and under-evaluated. Peribronchovascular involvement and lymph node enlargement are most frequent patterns. Interstitial disease pattern seems to be associated with a lower rate of treatment response. https:// bit.ly/3B54HiR

Cite this article as: Muller R, Habert P, Ebbo M, et al. Thoracic involvement and imaging patterns in IgG4-related disease. Eur Respir Rev 2021; 30: 210078 [DOI: 10.1183/16000617.0078-2021].

Copyright @The authors 2021

This version is distributed under the terms of the Creative Commons Attribution NonCommercial Licence 4.0. For commercial reproduction rights and permissions contact permissions@ersnet.org

This article has supplementary material available from err.ersjournals.com

Received: 18 March 2021 Accepted: 9 July 2021

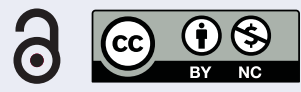

\section{Abstract}

Objective Immunoglobulin G4-related disease (IgG4-RD) is a rare orphan disease. Lung, pleura, pericardium, mediastinum, aorta and lymph node involvement has been reported with variable frequency and mostly in Asian studies. The objective of this study was to describe thoracic involvement assessed by high-resolution thoracic computed tomography (CT) in Caucasian patients with IgG4-RD.

Methods Thoracic CT scans before treatment were retrospectively collected through the French case registry of IgG4-RD and a single tertiary referral centre. CT scans were reviewed by two experts in thoracic imagery blinded from clinical data.

Results 48 IgG4-RD patients with thoracic involvement were analysed. All had American College of Rheumatology/European League Against Rheumatism classification scores $\geqslant 20$ and comprehensive diagnostic criteria for IgG4-RD. CT scan findings showed heterogeneous lesions. Seven patterns were observed: peribronchovascular involvement (56\%), lymph node enlargement (31\%), nodular disease (25\%), interstitial disease (25\%), ground-glass opacities (10\%), pleural disease (8\%) and retromediastinal fibrosis (4\%). In $37 \%$ of cases two or more patterns were associated. Asthma was significantly associated with peribronchovascular involvement $(\mathrm{p}=0.04)$. Among eight patients evaluated by CT scan before and after treatments, only two patients with interstitial disease displayed no improvement.

Conclusion Thoracic involvement of IgG4-RD is heterogeneous and likely underestimated. The main thoracic CT scan patterns are peribronchovascular thickening and thoracic lymph nodes.

\section{Introduction}

Immunoglobulin G4-related disease (IgG4-RD) is a systemic fibro-inflammatory disorder characterised by unifying histopathological findings [1]. Virtually all organs can be involved. In most case series, involvement of pancreas, bile ducts, salivary and lachrymal glands, retroperitoneum, lungs, kidneys, and aorta are reported with the highest frequencies [2-4]. Preferential clinical phenotypes have been suggested by cluster analysis [5]. Pulmonary involvement, usually from imaging studies, is reported in up to $14 \%$ of patients in large cohorts, with a higher frequency in patients with salivary gland and systemic involvement [6, 7]. 
In case series, the frequency of the different types of thoracic involvement is variable and the lesions observed are not specific for IgG4-RD. Lymph node enlargement is reported in $40-90 \%$ of patients, and pleura or lung involvement in 14-51\% [8-13]. However, only few case series have precisely described pulmonary and thoracic involvement, especially in non-Asian patients [14].

The aim of our study was to precisely describe thoracic computed tomography (CT) scan abnormalities in patients with well-characterised IgG4-RD, and to correlate the thoracic lesions and patterns observed with other organ involvements, respiratory disease such as asthma, and responses to treatment.

Materials and method

\section{Patients}

Patients with thoracic involvement were identified from the French case registry of IgG4-RD patients. This registry is the result of a multidisciplinary multicentric collaboration, which collects prospectively clinical, biological, radiological and pathological data from patients with IgG4-RD. When thoracic involvement was declared, physicians were asked to send the DICOM (Digital Imaging and Communications in Medicine) of CT scans performed at diagnosis before treatment (group 1, see Supplemental Figure S1). The case registry is authorised by the French authorities (Commission Nationale de l'Informatique et des Libertés). We also systematically reviewed all available thoracic CT scans from patients followed in a single tertiary centre without referenced thoracic involvement. All patients presenting with thoracic abnormalities were included in the study (group 2, Supplemental Figure S1). Only patients fulfilling both comprehensive diagnostic criteria and the American College of Rheumatology (ACR)/European League Against Rheumatism (EULAR) 2019 classification criteria (score $\geqslant 20$ ) were included in the study [4, 15]. Age, gender, time to diagnosis, organ involvements, biological evaluation, treatments and outcome were collected.

\section{Thoracic CT scan and reviewing methodology}

Patients from our centre underwent CT scan on the same system (Revolution EVO, GE Healthcare, WI, USA). CT scans were acquired in profound and maximal inspiration with the following parameters: detector collimation: $0.625 \mathrm{~mm}$; field of view: $500 \mathrm{~mm}$; matrix: 512×512; pitch: 1.375; gantry speed $0.35 \mathrm{~s} ; 120 \mathrm{Kv} ; 1 \mathrm{mAs} \cdot \mathrm{kg}^{-1}$ with care dose modulation, reconstruction in slices of thickness 1:1 mm. We applied dose modification manually according to the patient template, which means $140 \mathrm{kV}$ or $100 \mathrm{kV}$ if their weight were more than $120 \mathrm{~kg}$ or less than $60 \mathrm{~kg}$. All imaging data were reconstructed using high-resolution and standard algorithms. Concerning patients from the other centres, physicians were asked to send the anonymised DICOM of CT scans performed at diagnosis before treatment. All imported imageries included thin-section CT scans and high-resolution reconstruction filters. Subsequently, the system and the parameters were not available for imported CT. Monitors were used to view both mediastinal (width, $400 \mathrm{HU}$; level, $20 \mathrm{HU}$ ) and lung (width, $1600 \mathrm{HU}$; level, $-600 \mathrm{HU}$ ) windows. The pre-established top anatomic border was the lower part of the neck. The pre-established anatomic bottom boundary was the estimated location of the adrenal glands below the costophrenic angle. CT data were sent to an archiving and communicating system (Centricity Universal Viewer, GE Healthcare, WI, USA). All CT scans were reviewed by two thoracic radiologists blinded from clinical data and discrepancies were reviewed by the two radiologists together to reach a consensus.

Abnormalities were described according to the Fleischner glossary [16] as following: bronchiolitis (tree in bud or alveolar nodules), bronchiectasis, solid nodules, ground-glass opacities (GGO), areas of consolidation, pleural effusion or thickening, peribronchovascular thickening, mediastinal lymph nodes, mucoid impactions, septal thickening, honeycombing, reticulations, architectural distortion, and retromediastinal fibrosis (paravertebral soft tissue band). Subsequently, they were classified into four patterns as previously reported: nodular, round-shaped GGO, interstitial disease and peribronchovascular involvement [14]. Lymph node enlargement, pleural disease and retromediastinal fibrosis patterns were added to define a total of seven patterns of thoracic involvement (Supplemental Table S1). Nodular pattern was defined by the presence of multiple solid nodules; round-shaped GGO pattern by the presence of multiple GGO; interstitial disease pattern by the presence of septal thickening, honeycombing, reticulations or architectural distortion; peribronchovascular involvement pattern was defined by the presence of peribronchovascular thickening, bronchiolitis, area of consolidation or mucoid impaction; lymph node enlargement pattern by bilateral mediastinal lymph nodes; and pleural involvement pattern by pleural effusion or pleural thickening and retromediastinal fibrosis pattern by the presence of retromediastinal fibrosis. The specificity between thoracic involvement and IgG4-RD was also analysed according to the criteria of CORCORAN et al. [6]: thoracic biopsy-proved was necessary for "definite" IgG4-RD-related thoracic involvement; a typical imaging finding with no alternative explanation and response to treatment was defined as "highly probable" IgG4-RD-related thoracic involvement; typical radiology with no 
alternative explanation was defined "probable" IgG4-RD-related thoracic involvement; and radiology potentially consistent with IgG4-RD but with a plausible alternative cause was identified defined as "possible” IgG4-RD-related thoracic involvement. Response to treatment of thoracic lesions was defined by CT scan comparative analysis before and after treatment: complete response was retained if all thoracic abnormalities disappeared after treatment, no response was retained if thoracic abnormalities remained unchanged and partial response was retained in all cases with improvement of thoracic lesions without complete disappearance.

\section{Statistical analysis}

Quantitative variables were expressed as mean or median, standard deviation and range. Qualitative variables were expressed as numbers, proportions and percentages. The differences between groups were compared by the chi-square or Fisher's exact tests for qualitative variables, and by the Mann-Whitney test for quantitative variables. A $\mathrm{p}<0.05$ was considered significant.

\section{Results}

\section{Patients' characteristics}

At the time of the study, 222 patients were included in the French national cohort of IgG4-RD (Supplemental Figure S1). A thoracic involvement was notified in 56 of them (25\%), among whom CT scans at diagnosis could be collected in 28 (50\%) cases (group 1). We also systematically reviewed the CT scans from 26 patients without notified thoracic involvement followed in our tertiary referral centre. This analysis revealed thoracic abnormalities in 20/26 IgG4-RD patients (77\%) (group 2).

After pooling patients from groups 1 and 2, a total of 48 patients with CT scan thoracic involvement were analysed (table 1). All patients fulfilled ACR/EULAR classification criteria with a score $\geqslant 20$ (range: 25-75) [4], even if six patients (13\%) presented with eosinophilia $>3000$ cells $\cdot \mathrm{mm}^{-3}$. Mean age was $60 \pm 15$ years, with male predominance (81\%). Nine patients (19\%) were smokers or former smokers, 18 (37\%) reported atopy, and one was exposed to asbestos. Respiratory symptoms were reported in 14 patients and consisted

TABLE 1 Clinical and biological characteristics of 48 patients with immunoglobulin G4-related disease (IgG4-RD) thoracic involvement

Characteristic

Patients $(n=48)$

\begin{tabular}{|c|c|}
\hline \multicolumn{2}{|l|}{ Demographic data } \\
\hline Male & $39(81)$ \\
\hline Median age at onset (years) & $60(23-83)$ \\
\hline Median diagnostic delay (months) & $6(1-240)$ \\
\hline Smoker or former smoker & $9(19)$ \\
\hline History of atopy & $18(37)$ \\
\hline \multicolumn{2}{|l|}{ Organ involvement of IgG4-RD } \\
\hline Median number of organs affected & $3(1-6)$ \\
\hline Lymph nodes & $30(62.5)$ \\
\hline Pancreatitis & $15(31.2)$ \\
\hline Tubulointerstitial nephritis & $15(31.2)$ \\
\hline Sialadenitis & $15(31.2)$ \\
\hline Retroperitoneal fibrosis & $10(20.8)$ \\
\hline Sclerosing cholangitis & $10(20.8)$ \\
\hline Aortitis & $8(16.6)$ \\
\hline Dacryoadenitis & $6(12.5)$ \\
\hline Prostatitis & $3(6.2)$ \\
\hline \multicolumn{2}{|l|}{ Biological characteristics } \\
\hline Elevated serum IgG $\left(>1400 \mathrm{mg} \cdot \mathrm{dL}^{-1}\right)$ & $43(89.5)$ \\
\hline Elevated serum IgG4 (>135 $\left.\mathrm{mg} \cdot \mathrm{dL}^{-1}\right)$ & $43(89.5)$ \\
\hline Elevated serum IgG4 >2xN & $32(66.7)$ \\
\hline Median serum IgG4 level $\left(\mathrm{mg} \cdot \mathrm{dL}^{-1}\right)$ & $430(23-2040)$ \\
\hline Hypocomplementaemia & $13(27)$ \\
\hline Elevated CRP (>5 mg. $\left.\mathrm{L}^{-1}\right)$ & $39(81.2)$ \\
\hline Positive ANA (>1/160) & $9(18.8)$ \\
\hline
\end{tabular}


of wheezing (64\%), cough (21\%), thoracic pain $(14 \%)$ or dyspnoea $(14 \%)$. Thoracic involvement was reported at diagnosis in 13 patients (27\%), and during follow-up (at a relapse) in 34 patients (72\%). The relapses occurred during maintenance therapy in 19 patients and in the absence of any treatment in the others.

Most patients presented with systemic involvement, defined by three or more organs involved (63\%, mean $3 \pm 1.5$ organs involved). Isolated thoracic involvement with histological confirmation was observed in only one patient (figure 1). The main extra-thoracic associated organ involvements consisted of lymph nodes (62\%), pancreas $(31 \%)$, salivary glands $(31 \%)$, kidneys $(31 \%)$, bile ducts $(21 \%)$ and retroperitoneum (21\%). The mean serum IgG4 level was $700 \pm 680 \mathrm{mg} \cdot \mathrm{dL}^{-1}$, median $425 \mathrm{mg} \cdot \mathrm{dL}^{-1}$. Hypocomplementaemia was reported in $27 \%$ of patients, and elevated serum $C$ reactive protein (CRP) level $\left(>5 \mathrm{mg} \cdot \mathrm{L}^{-1}\right)$ in $81 \%$. Hypereosinophilia $>1500$ cells $\cdot \mathrm{mm}^{-3}$ was observed in 11 patients $(22 \%)$, ranging from $1600 \mathrm{cells} \cdot \mathrm{mm}^{-3}$ to 18000 cells $\cdot \mathrm{mm}^{-3}$. The six patients with hypereosinophilia $>3000$ cells $\cdot \mathrm{mm}^{-3}$ were maintained in the study because they presented with definite IgG4-RD and no other diagnosis related to eosinophilia.

\section{Thoracic imaging features}

CT features of IgG4-RD thoracic involvement are presented in Supplemental Table S1. Lung involvement, defined as peribronchovascular thickening, GGO, nodular or interstitial disease pattern, was present in 43/ 48 patients (89\%). Peribronchovascular thickening and lymph nodes were observed in 54\% of patients. When thoracic abnormalities were classified by patterns, peribronchovascular involvement pattern (56\%) and lymph node enlargement pattern (31\%) were the most frequent. Nodular and interstitial disease patterns were present in 25\%. GGO, pleural disease and retromediastinal fibrosis patterns were less common in $10 \%, 8 \%$ and $4 \%$ of patients, respectively. 18 patients (37\%) had at least two types of thoracic involvement patterns (table 2). The peribronchovascular involvement pattern was isolated in 13/27 cases (48\%), while the nodular pattern in 3/7 cases (43\%), the interstitial disease pattern in 2/7 cases (29\%) and the lymph node enlargement pattern in 3/15 cases (20\%). GGO and pleural disease patterns were never isolated. Both patients with a retro-mediastinal fibrosis pattern (paravertebral soft-like band) had no other thoracic involvement. No patient had pericardial involvement on thoracic CT.
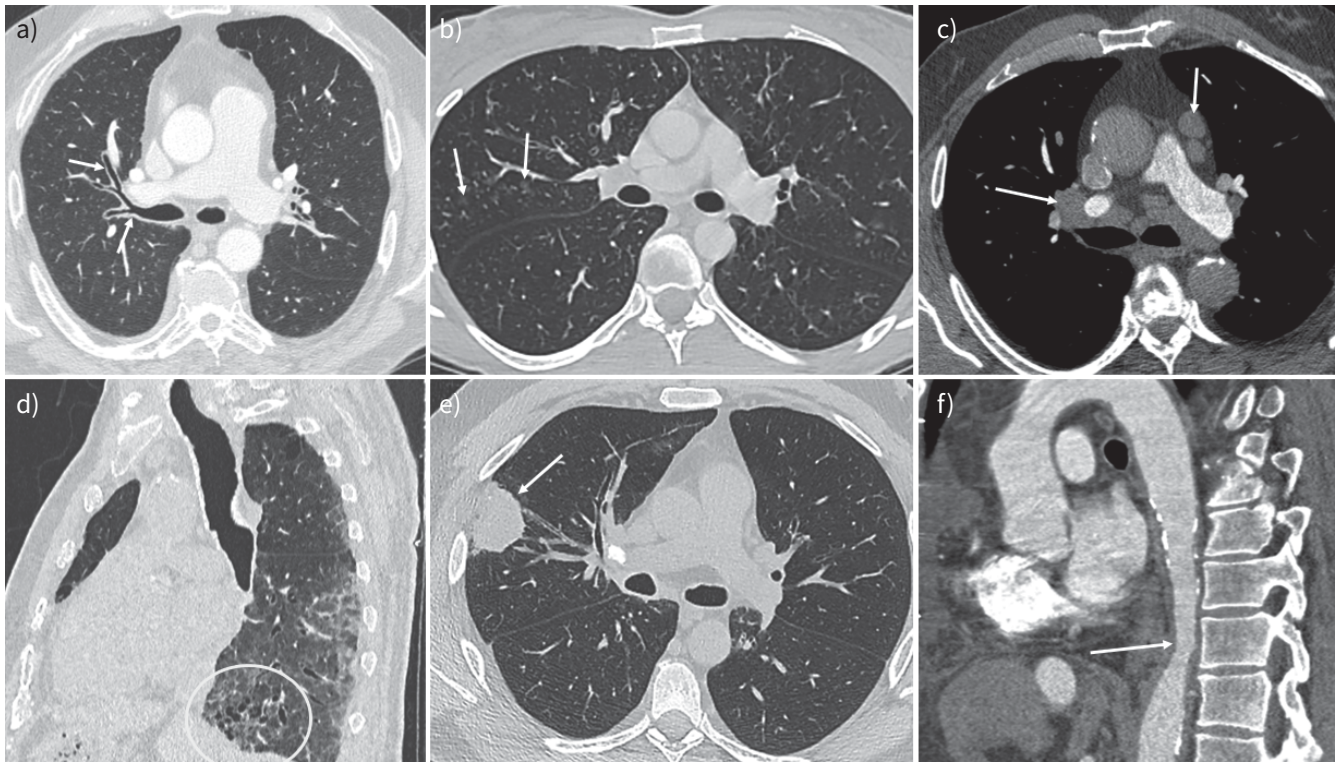

FIGURE 1 Various imaging patterns of immunoglobulin G4-related disease thoracic involvement (each computed tomography (CT) scan section comes from a different patient). a) Axial contrast-enhanced thoracic CT scan showing an important bronchial thickening of the segmental right superior lobe bronchi (arrows). b) Axial unenhanced thoracic CT scan showing nodular ground-glass opacities in the posterior segment of the right superior lobe (arrows). c) Axial enhanced thoracic CT scan showing lymph nodes enlargement in the mediastinum in situation 5 and $10 R$ (arrows). d) Sagittal reconstruction of thoracic CT scan showing distal bronchial distension and traction bronchiectasis in the interstitial lung pattern (circle). e) Axial unenhanced thoracic CT scan showing a mass in the right superior lobe (arrow). f) Sagittal enhanced aortic CT scan showing a stenosis of descending aorta and aortic wall thickening due to posterior mediastinitis (arrow). 
TABLE 2 Association between immunoglobulin G4-related disease (IgG4-RD) thoracic involvement patterns.

\begin{tabular}{|c|c|c|c|c|c|c|}
\hline Nodular & $\begin{array}{l}\text { Interstitial } \\
\text { disease }\end{array}$ & $\begin{array}{l}\text { Peribroncho } \\
\text { vascular } \\
\text { involvement }\end{array}$ & $\begin{array}{c}\text { Ground-glass } \\
\text { opacities }\end{array}$ & $\begin{array}{l}\text { Lymph node } \\
\text { enlargement }\end{array}$ & $\begin{array}{c}\text { Retro } \\
\text { mediastinal } \\
\text { fibrosis }\end{array}$ & $\begin{array}{l}\text { Pleural } \\
\text { disease }\end{array}$ \\
\hline
\end{tabular}

\begin{tabular}{|c|c|c|c|c|c|c|c|}
\hline Nodular & 7 & & & & & & \\
\hline Interstitial disease & 1 & 7 & & & & & \\
\hline $\begin{array}{l}\text { Peribronchovascular } \\
\text { involvement }\end{array}$ & 2 & 3 & 27 & & & & \\
\hline $\begin{array}{l}\text { Lymph node } \\
\text { enlargement }\end{array}$ & 3 & 3 & 9 & 0 & 15 & & \\
\hline Retromediastinal fibrosis & 0 & 0 & 0 & 0 & 0 & 2 & \\
\hline
\end{tabular}

Causal relationship between CT scan lesion and IgG4-RD

According to the criteria of CoRCoran et al. [6] (table 3), we classified 23 patients (48\%) as definitive, nine (19\%) as highly probable, 15 (31\%) as probable and one (2\%) with possible involvement. No differences in thoracic involvement pattern, age, gender, serum IgG4 level, history of smoking or exposure to asbestos were observed between groups. Histologic features of the 23 patients with definitive involvement are shown in Supplemental Table S2.

Correlations of thoracic CT scan lesions with IgG4-RD characteristics

Characteristics of patients classified in the seven different CT scan patterns (nodular, interstitial disease, peribronchovascular involvement, round-shaped GGO, lymph node enlargement, pleural disease and

TABLE 3 Classification of immunoglobulin G4-related disease (IgG4-RD) thoracic involvement according to the criteria of CoRCORAN et al. [6]

\begin{tabular}{|c|c|c|c|c|c|c|c|}
\hline $\begin{array}{l}\text { Category of thoracic } \\
\text { involvement }\end{array}$ & $\begin{array}{l}\text { Number of } \\
\text { patients } \\
\text { (group 1/ } \\
\text { group2) }\end{array}$ & Thoracic patterns & $\begin{array}{l}\text { Age at } \\
\text { diagnosis, } \\
\text { years } \\
\text { median } \pm \text { SD }\end{array}$ & $\begin{array}{c}\text { Male } \\
\mathrm{n} / \mathrm{N}(\%)\end{array}$ & $\begin{array}{l}\text { Elevated } \\
\text { serum IgG4 } \\
\text { level } \\
\mathrm{n} / \mathrm{N}(\%)\end{array}$ & $\begin{array}{c}\text { Smoking } \\
\text { history }>10 \\
\text { pack years } \\
\mathrm{n} / \mathrm{N}(\%)\end{array}$ & $\begin{array}{l}\text { Industrial or } \\
\text { environmental } \\
\text { exposure to } \\
\text { asbestos } \\
\mathrm{n} / \mathrm{N}(\%)\end{array}$ \\
\hline $\begin{array}{l}\text { Definite } \\
\text { Biopsy proved } \\
\text { thoracic involvement }\end{array}$ & $23 / 48$ & $\begin{array}{c}15 \text { lymph node } \\
\text { enlargement } \\
13 \text { peribronchovascular } \\
\text { involvements } \\
\text { Seven nodular } \\
\text { Four interstitial disease } \\
\text { Two GGO }\end{array}$ & $59 \pm 14$ & $\begin{array}{c}20 / 23 \\
(87)\end{array}$ & $\begin{array}{c}20 / 23 \\
(87)\end{array}$ & $\begin{array}{l}3 / 23 \\
(13)\end{array}$ & $\begin{array}{c}0 / 23 \\
(0)\end{array}$ \\
\hline $\begin{array}{l}\text { Highly probable } \\
\text { Typical radiology } \\
\text { with no alternative } \\
\text { explanation and } \\
\text { response to } \\
\text { treatment }\end{array}$ & $9 / 48$ & $\begin{array}{c}\text { Seven } \\
\text { peribronchovascular } \\
\text { involvement } \\
\text { Four lymph node } \\
\text { enlargement } \\
\text { Three GGO } \\
\text { One nodular } \\
\text { One interstitial disease }\end{array}$ & $49 \pm 17$ & $\begin{array}{l}8 / 9 \\
(89)\end{array}$ & $\begin{array}{l}8 / 9 \\
(89)\end{array}$ & $\begin{array}{l}1 / 9 \\
(11)\end{array}$ & $\begin{array}{l}0 / 9 \\
(0)\end{array}$ \\
\hline $\begin{array}{l}\text { Probable } \\
\text { Typical radiology } \\
\text { with no alternative } \\
\text { explanation }\end{array}$ & $15 / 48$ & $\begin{array}{c}\text { Eight } \\
\text { peribronchovascular } \\
\text { involvements } \\
\text { Three lymph node } \\
\text { enlargement } \\
\text { Three nodular }\end{array}$ & $65 \pm 16$ & $\begin{array}{c}10 / 15 \\
(66)\end{array}$ & $\begin{array}{c}14 / 15 \\
(93)\end{array}$ & $\begin{array}{l}3 / 15 \\
(20)\end{array}$ & $\begin{array}{l}0 / 15 \\
(0)\end{array}$ \\
\hline $\begin{array}{l}\text { Possible } \\
\text { Radiology potentially } \\
\text { consistent with } \\
\text { IgG4-RD but plausible } \\
\text { alternative cause } \\
\text { identified }\end{array}$ & $1 / 48$ & One interstitial disease & 76 & $\begin{array}{c}1 / 1 \\
(100)\end{array}$ & $\begin{array}{c}1 / 1 \\
(100)\end{array}$ & $\begin{array}{l}0 / 1 \\
(0)\end{array}$ & $\begin{array}{c}1 / 1 \\
(100)\end{array}$ \\
\hline
\end{tabular}


TABLE 4 Clinico-biological characteristics of patients according to the immunoglobulin G4-related disease (IgG4-RD) thoracic pattern.

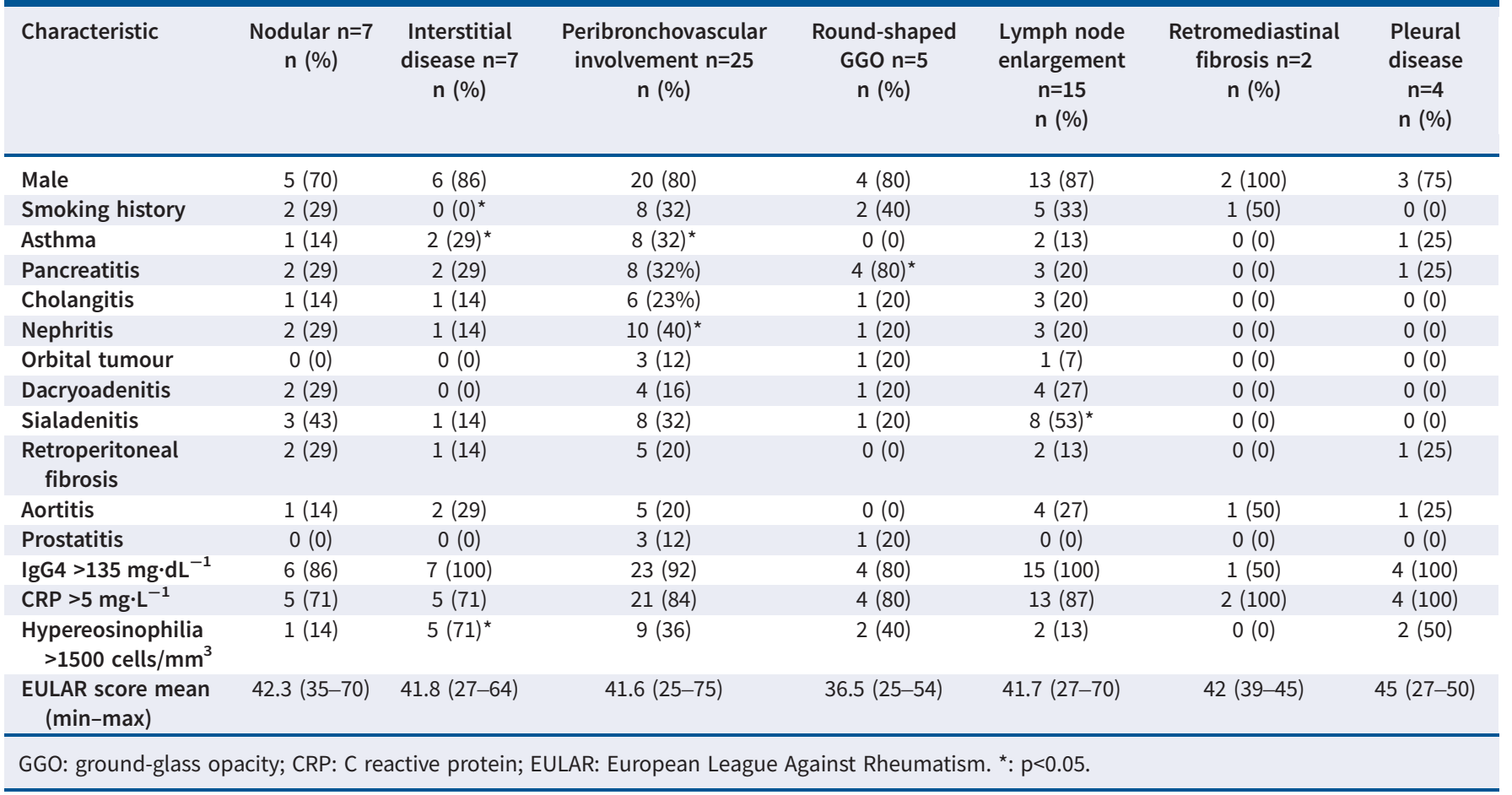

retromediastinal fibrosis) groups are presented in table 4. Asthma, defined from medical records, was found associated with peribronchovascular involvement pattern $(\mathrm{p}=0.041)$. The round-shaped GGO pattern was associated with IgG4-related pancreatitis $(\mathrm{p}=0.013)$. The lymph node enlargement pattern was associated with sialadenitis $(\mathrm{p}=0.041)$. Hypereosinophilia was associated with the interstitial disease pattern $(\mathrm{p}=0.031)$.

\section{Response to treatment of IgG4-RD thoracic involvement}

Eight patients underwent multiple longitudinal evaluations with a thoracic CT scan with thin sections before and after treatment. The mean time of follow-up was 30 months (from 6 to 73 months). Patterns of IgG4-RD thoracic involvement before treatment were: nodular $(n=1)$, interstitial disease $(n=2)$, peribronchovascular involvement $(n=4)$, GGO $(n=2)$, retromediastinal fibrosis $(n=1)$ and lymph node enlargement $(n=1)$. Patients were treated for IgG4-RD by prednisone $(n=2)$, rituximab $(n=1)$, prednisone and rituximab $(n=4)$ or prednisone and azathioprine $(n=1)$. Complete remission of thoracic abnormalities was observed in two patients treated with prednisone or prednisone and rituximab (Supplemental Figure S2). Partial remission was observed in four patients treated with rituximab $(n=1)$, prednisone and rituximab $(n=2)$, and prednisone and azathioprine $(n=1)$, respectively. In two patients with an interstitial disease pattern treated with prednisone or prednisone and rituximab, their lesions remained unchanged.

\section{Discussion}

We describe thoracic CT scan abnormalities and IgG4-RD thoracic involvement patterns in 48 IgG4-RD patients fulfilling ACR/EULAR classification criteria [4, 15]. To our knowledge, this is the largest series reported to date. Almost half of the patients $(n=23)$ had a thoracic biopsy (lymph nodes, lung or pleura). Pathological confirmation of IgG4-RD was obtained from extra-thoracic organ biopsies in others and all these patients presented with otherwise typical IgG4-RD involvement.

The most frequent lesions observed were peribronchovascular thickening (54\%) and mediastinal lymph nodes (54\%). The other thoracic lesions were: solid nodules (33\%), areas of consolidation (25\%), bronchiectasis (19\%), bronchiolitis (15\%), septal thickening (15\%), reticulations (15\%) and GGO (13\%) (Supplemental Table S1). Pleural effusion or thickening and mediastinal fibrosis were less frequent than lung involvement. Further classification by patterns, according to predominant thoracic abnormalities, found that peribronchovascular involvement (56\%) and lymph node enlargement (31\%) patterns were the most frequent [14]. All patients, except the two with mediastinal fibrosis, presented more than one pattern (table 2). 
We found no preferential association within different patterns. In addition, there were no major differences in gender, age, IgG4 level, asbestosis or tobacco exposure between patterns. We confirm as reported from other series that lung involvement in IgG4-RD is heterogeneous [6, 7, 11-14, 17-20]. The discrepancies with other series between the frequencies of lung abnormalities observed suggests either a bias related to retrospective data collection, the lack of central imagery reviewing in some studies or a role of ethnic background. SARAYA et al. [21] reported GGO in 69\% $(\mathrm{n}=16)$ compared to only $13 \%$ in our series. The study from Kang et al. [22] ( $n=37$ ) with a classification in five radiologic subtypes found that solid nodular and alveolar consolidative were the most frequent (30\% each). None of these patterns are specific for IgG4-RD. However, we could suggest that the association of two or more patterns in a patient (observed in $37 \%$ of patients of this series) should be considered as an argument in favour of IgG4-RD [23].

IgG4-RD should be evoked frequently in cases of lung CT scan abnormalities, especially in the assessment of multisystem disease. A complete patient workup including the search for other organ involvement and biological and pathological assessment should be always considered. Other lung diseases need to be excluded because of the lack of specificity and the diversity of patterns. The patients in this study had a long follow-up and extensive diagnostic workup, responding to the ACR/EULAR criteria. The exposition to tobacco use $(19 \%)$ or occupational or environmental toxicants $(2 \%)$ were not frequent. When we categorised the patients based on the probability of lung involvement related to IgG4-RD as done by Corcoran et al. [6], 47/48 had at least probable involvement (table 3). This make us confident that the lesions observed here, before any treatment, were related to IgG4-RD. Asthma, hypereosinophilia and absence of smoking were significantly associated with interstitial disease pattern. Atopy or asthma has a close relationship to IgG4-RD and is reported in one third of IgG4-RD patients [2, 24, 25]. Of note in our study, six patients had an eosinophil count $\geqslant 3000$ cells $\cdot \mathrm{mm}^{-3}$, which is considered as an exclusion criteria in the ACR/EULAR classification. We believe that these presented with bona fide IgG4-RD in the absence of an alternative diagnosis. These patients, as others, presented with various patterns: peribronchovascular involvement 4/6, interstitial disease 2/6, GGO 2/6 and lymph node enlargement 2/6.

This wide range of lung lesions in IgG4-RD suggests various pathophysiological mechanisms. Interestingly, pathological findings in the lungs are slightly different from those reported in other organs in IgG4-RD with inflammation of the interstitium and alveolar space, sometimes obliterative arteritis and less frequently storiform fibrosis or obliterative phlebitis $[1,12,26]$. The pattern of fibrosis in the lung is less storiform than reported in other organs and associated with fibroblast proliferation. Studies of broncho-alveolar lavage fluid reported lymphocytosis [11]. The lesions revealed by CT scans confirm that some lesions in IgG4RD, as in sarcoidosis (thickening of the perilymphatic interstitium, thoracic lymph nodes, peribronchovascular and pleural involvement), are related to the involvement of the lymphatic system [27]. Some patterns were associated here with particular extra-thoracic organ manifestations: GGO pattern and pancreatitis, peribronchovascular involvement pattern and nephritis, interstitial disease pattern, and eosinophilia and lymph nodes enlargement pattern with sialadenitis (table 4). Such associations on a limited case series are weak but could mirror variations observed in pathological lesions in between organs in the disease [28]. We compared the phenotype of organ involvement and biological variables from the 48 patients with patients from the case registry without thoracic involvement $(n=166)$ (Supplemental Table S3). The only differences observed were a higher number of organs involved (3.04 versus 2.53, $\mathrm{p}=0.04$ ), higher serum CRP levels $\left(\mathrm{CRP}>5 \mathrm{mg} \cdot \mathrm{L}^{-1}, 73 \%\right.$ versus $\left.81 \%, \mathrm{p}<0.02\right)$ and hypergammaglobulinemia $(89 \%$ versus $70.3 \%, \mathrm{p}<0.01)$ suggesting an ongoing systemic form of the disease. However, as will be discussed later, this comparison is hampered by the lack of systematic review of CT scans from patients of the registry outside of our centre.

Assessing the diagnosis of IgG4-RD lung involvement is important because patients usually respond to steroid therapy. As discussed above, the patterns observed may represent different pathological mechanisms and therefore the response to treatment or the risk of relapse could vary depending on the type. This has been shown for instance for interstitial lung disease in systemic sclerosis classified in non-specific interstitial pneumonia or usual interstitial pneumonia, both reported in IgG4-RD [27]. The first pattern is more frequent but the second is associated with aggressive interstitial lung disease with a poorer outcome [29]. We evaluated CT scans before and after treatment in eight cases and observed that only interstitial disease pattern did not improve. Kang et al. [22] reported that alveolar interstitial type showed the lowest response rate. Of note, Corcoran et al. [6] reported from their series that in 4/7 patients who died thoracic pathology was recorded on the death certificate. IgG4-RD thoracic involvement was not responsible for death in all these cases but it suggests that lung involvement should be carefully investigated in IgG4-RD.

We were surprised by the high frequency of thoracic involvement observed after expert review of CT scans from patients for which thoracic involvement was not considered at the initial IgG4-RD evaluation. The review of CT scans from 26 patients (without initially recorded thoracic abnormalities) identified lesions in 
20 of them (76\%, group 2). Lesions un-noticed previously were mediastinal lymph nodes (55\%), peribronchovascular thickening (50\%) and bronchial abnormalities (bronchiectasis or bronchiolitis, 25\%). Conversely, patients with GGO, pleural involvement or retromediastinal fibrosis were well diagnosed as having thoracic involvement from the first CT scan evaluation. This suggests a high frequency of thoracic involvement in IgG4-RD probably being under-evaluated in case series without systematic imagery reviews $[6,14]$. The specific focus on thoracic imagery by experts and the production of thin CT slices in this study probably account for this frequency.

From this study, we think that it is useful to evaluate thoracic CT scans when IgG4-RD is suspected. At the very least, we suggest that the thoracic CT scans frequently performed at initial staging of the disease should be carefully investigated. Identifying peribronchovascular thickening and soft band-like tissues, considered as more specific, will help diagnoses according to ACR/EULAR classification criteria. Multiple organ involvement in IgG4-RD is a risk factor for relapses, therefore identifying thoracic involvement can have an impact on prognosis. We do not think that low-dose CT scans are a valuable tool in IgG4-RD because the low quality of soft tissue analyses could miss small GGO lesions and interstitial lung abnormalities, do not allow accurate evaluation of bronchial wall thickening and can underestimate mediastinal lymph node involvement. We also suggest that, because some abnormalities reported are subtle and could be easily missed, CT scans should be reviewed by specialist radiologists with expertise in the field. In this study, we did not analyse 18-fluorodeoxyglucose (18FDG) positron emission tomography (PET) CT, which has been shown to be a reliable technique for assessing IgG4-RD activity and detect clinically asymptomatic IgG4-RD organ involvement in the thorax (lung or pleura) [30]. PET CT is also useful to assess treatment response but clinical-radiological discrepancies with 18FDG uptake have been reported for lung lesions and lymph nodes [31].

This study has some limitations, mainly related to the retrospective collection of data and to the lack of systematic pathological documentation of lung involvement. Moreover, lung functional tests were lacking and only a few patients could be analysed after treatment. Not all CT scans from the whole case registry were retrospectively reviewed and therefore the comparison between patients with and without thoracic involvement could be biased. Because the inclusion criteria required an ACR/EULAR score $>20$ we probably missed patients with definite isolated lung involvement. Their absence in the analysis focusing mainly on patients with multiorgan involvement introduces a potential bias in our analysis.

Strengths of this study are the central and systematic reviews by experts of thin-section thoracic CT scans from patients characterised by the recent IgG4-RD ACR/EULAR classification criteria.

\section{Conclusion}

The thoracic and, in particular, lung involvement of IgG4-RD is heterogeneous and probably under-evaluated. Peribronchovascular involvement and lymph node enlargement are the most frequent patterns. Nodular disease, interstitial disease, GGO, pleural disease and mediastinal fibrosis are less frequent. None of these patterns are specific. The interstitial disease pattern seems to be associated with a lower rate of treatment response and both interstitial disease and peribronchovascular involvement patterns are associated with asthma.

Provenance: Submitted article, peer reviewed.

Conflict of interest: D. Launay reports grants or contracts from Servier paid to University of Lille, and consulting fees from Biocrist and Boeringer-Ingelheim, outside the submitted work. The remaining authors declare no conflict of interest.

\section{References}

1 Deshpande V, Zen Y, Chan JK, et al. Consensus statement on the pathology of IgG4-related disease. Mod Pathol 2012; 25: 1181-1192.

2 Stone JH, Zen Y, Deshpande V. IgG4-related disease. N Engl J Med 2012; 366: 539-551.

3 Ebbo M, Schleinitz N. IgG4-related disease (IgG4-RD). Ann Pathol 2012; 32: S70-S71.

4 Wallace ZS, Naden RP, Chari S, et al. The 2019 American College of Rheumatology/European League Against Rheumatism classification criteria for IgG4-related disease. Ann Rheum Dis 2020; 79: 77-87.

5 Wallace ZS, Zhang Y, Perugino CA, et al. Clinical phenotypes of IgG4-related disease: an analysis of two international cross-sectional cohorts. Ann Rheum Dis 2019; 78: 406-412.

6 Corcoran JP, Culver EL, Anstey RM, et al. Thoracic involvement in IgG4-related disease in a UK-based patient cohort. Respir Med 2017; 132: 117-121. 
7 Morales AT, Cignarella AG, Jabeen IS, et al. An update on IgG4-related lung disease. Eur J Intern Med 2019; 66: 18-24.

8 Nakanuma Y, Zen Y, Harada K, et al. Application of a new histological staging and grading system for primary biliary cirrhosis to liver biopsy specimens: Interobserver agreement. Pathol Int 2010; 60: 167-174.

9 Hamano H, Tanaka E, Ishizaka N, et al. IgG4-related disease - a systemic disease that deserves attention regardless of one's subspecialty. Intern Med 2018; 57: 1201-1207.

10 Nakazawa T, Naitoh I, Ando T, et al. A case of advanced-stage sclerosing cholangitis with autoimmune pancreatitis not responsive to steroid therapy. JOP J Pancreas 2010; 11: 58-60.

11 Tsushima K, Tanabe T, Yamamoto $\mathrm{H}$, et al. Pulmonary involvement of autoimmune pancreatitis. Eur J Clin Invest 2009; 39: 714-722.

12 Zen Y, Inoue D, Kitao A, et al. IgG4-related lung and pleural disease: a clinicopathologic study of 21 cases. Am J Surg Pathol 2009; 33: 1886-1893.

13 Fujinaga $\mathrm{Y}$, Kadoya M, Kawa S, et al. Characteristic findings in images of extra-pancreatic lesions associated with autoimmune pancreatitis. Eur J Radiol 2010; 76: 228-238.

14 Inoue D, Zen $\mathrm{Y}$, Abo $\mathrm{H}$, et al. Immunoglobulin G4-related lung disease: CT findings with pathologic correlations. Radiology 2009; 251: 260-270.

15 Umehara H, Okazaki K, Masaki Y, et al. Comprehensive diagnostic criteria for IgG4-related disease (IgG4-RD), 2011. Mod Rheumatol 2012; 22: 21-30.

16 Hansell DM, Bankier AA, MacMahon $\mathrm{H}$, et al. Fleischner society: glossary of terms for thoracic imaging. Radiology 2008; 246: 697-722.

17 Ogoshi T, Kido T, Yatera K, et al. Incidence and outcome of lung involvement in IgG4-related autoimmune pancreatitis. Respirology 2015; 20: 1142-1144.

18 Lv X, Gao F, Liu Q, et al. Clinical and pathological characteristics of IgG4-related interstitial lung disease. Exp Ther Med 2018; 15: 1465-1473.

19 Sekiguchi H, Horie R, Kanai M, et al. IgG4-related disease: retrospective analysis of one hundred sixty-six patients. Arthritis Rheumatol Hoboken NJ 2016; 68: 2290-2299.

20 Fei $\mathrm{Y}$, Shi J, Lin W, et al. Intrathoracic involvements of immunoglobulin G4-related sclerosing disease. Medicine 2015; 94: e2150.

21 Saraya T, Ohkuma K, Fujiwara M, et al. Clinical characterization of 52 patients with immunoglobulin G4-related disease in a single tertiary center in Japan: special reference to lung disease in thoracic high-resolution computed tomography. Respir Med 2017; 132: 62-67.

22 Kang J, Park S, Chae EJ, et al. Long-term clinical course and outcomes of immunoglobulin G4-related lung disease. Respir Res 2020; 21: 273.

23 Campbell SN, Rubio E, Loschner AL. Clinical review of pulmonary manifestations of IgG4-related disease. Ann Am Thorac Soc 2014; 11: 1466-1475.

24 Zhang W, Stone JH. Management of IgG4-related disease. Lancet Rheumatol 2019; 1: e55-e65.

25 Marie BDS, Urban ML, Vély F, et al. Pathophysiology of IgG4-related disease: a $T$ follicular helper cells disease? Presse Medicale 2020; 49: 104013.

26 Shrestha B, Sekiguchi H, Colby TV, et al. Distinctive pulmonary histopathology with increased IgG4-positive plasma cells in patients with autoimmune pancreatitis: report of 6 and 12 cases with similar histopathology. Am J Surg Pathol 2009; 33: 1450-1462.

27 Ryu JH, Horie R, Sekiguchi H, et al. Spectrum of disorders associated with elevated serum IgG4 levels encountered in clinical practice. Int J Rheumatol 2012; 2012: 232960.

28 Deshpande V, Sainani NI, Chung RT, et al. IgG4-associated cholangitis: a comparative histological and immunophenotypic study with primary sclerosing cholangitis on liver biopsy material. Mod Pathol 2009; 22: 1287-1295.

29 Perelas A, Silver RM, Arrossi AV, et al. Systemic sclerosis-associated interstitial lung disease. Lancet Respir Med 2020; 8: 304-320.

30 Ebbo M, Grados A, Guedj E, et al. Usefulness of 2-[18F]-fluoro-2-deoxy-D-glucose-positron emission tomography/computed tomography for staging and evaluation of treatment response in IgG4-related disease: a retrospective multicenter study. Arthritis Care Res 2014; 66: 86-96.

31 Berti A, Della-Torre E, Gallivanone F, et al. Quantitative measurement of 18F-FDG PET/CT uptake reflects the expansion of circulating plasmablasts in IgG4-related disease. Rheumatol Oxf Engl 2017; 56: 2084-2092. 